\title{
Scaling of the Microrheology of Semidilute F-Actin Solutions
}

\author{
T. Gisler* and D. A. Weitz \\ Department of Physics, University of Pennsylvania, 209 S. 33rd Street, Philadelphia, Pennsylvania 19104
}

(Received 5 October 1998)

\begin{abstract}
The viscoelasticity of actin networks is probed over an extended range of frequencies using microrheology techniques, where the thermal motion of small beads in the network is measured using diffusing-wave spectroscopy. Despite large sample-to-sample variations, the data exhibit an unexpected scaling behavior and can all be collapsed onto a single master curve, indicative of a surprising universality in the elastic properties. The scaled data provide a precise measure of the average behavior of the actin networks and indicate that at high frequencies $\omega$, the shear modulus, increases as $\omega^{3 / 4}$.
\end{abstract}

PACS numbers: 87.19.Tt, 61.25.Hq, 83.10.Nn, 83.50.Fc

Eukaryotic cells owe their mechanical strength mainly to the cytoskeleton, an intricate network of filamentous proteins such as tubulin, vimentin, spectrin, and actin [1]. Filamentous or F-actin, the most abundant of these cytoskeletal proteins, forms by the polymerization of actin monomers in the presence of $\mathrm{K}^{+}$or $\mathrm{Mg}^{2+}$, resulting in a semiflexible polymer that can be many microns long. Its persistence length, $l_{\mathrm{p}}$, has been measured to be about 10-20 $\mu \mathrm{m}$ [2-4], about 3 orders of magnitude larger than the filament diameter, $d=7 \mathrm{~nm}$ [5]. Because of this large aspect ratio, actin filaments form semidilute solutions at extremely low volume fractions $\varphi \sim\left(d / l_{\mathrm{p}}\right)^{2}$, with an elastic modulus orders of magnitude larger than that of flexible polymers at the same volume fraction. A theoretical understanding of this elasticity is still controversial; very different results are predicted using effective medium models [6], models based on mechanical networks $[7,8]$, networks of semiflexible molecules with effectively permanent cross-links [9], and models which consider the unique properties of networks, at different concentrations, of semiflexible polymers [6,10-13]. Experimental measures of the modulus also suffer from wide variations, with reported values of the frequency dependent elastic modulus, $G^{\prime}(\omega)$, varying by as much as 2 orders of magnitude for apparently identical samples [14]. While the origin of this variation is not fully resolved, it is likely a combined effect caused by the variation in the filament length distribution and by the presence of small quantities of proteins which can act as chemical cross-links, leading to dramatic, but random, changes in the modulus. A definitive test of the proposed theoretical models requires a highly purified sample with carefully controlled filament length distribution [15]. However, it is perhaps even more crucial to develop a better understanding of the behavior of the actin networks more typically produced, both to better comprehend their properties and to determine the origin of the variability.

One feature of the elastic response of actin networks that should transcend the difficulty in obtaining reproducible shear moduli is the behavior at high frequencies.
When the wavelength of elastic excitation is shorter than the average spacing between entanglements, the modulus should be sensitive only to properties of the actin filaments themselves. However, even here, there are competing predictions; simple considerations for the dispersion of bending modes of rigid rods suggest that the complex shear modulus should scale as $G^{*}(\omega) \sim \omega^{3 / 4}[12,13,16]$, while other considerations of the longitudinal relaxation of a single filament suggest a contribution with a more rapid variation, $G^{*}(\omega) \sim \omega^{7 / 8}$ [17]. Unfortunately, however, the frequencies at which this behavior should be observed are somewhat beyond those accessible with traditional mechanical rheometers. Instead, the high frequency behavior is probed using what has come to be called microrheology; a small probe particle is embedded in the gel, and its frequency dependent response is determined either by forcing its motion with magnetic beads in an applied field $[16,18,19]$ or by monitoring its thermally induced motion [20-22]. If the particle is large compared to the average mesh size, $l_{\mathrm{m}}$, its response should reflect the average rheological properties of the surrounding medium. However, results reported to date rarely reveal a low-frequency plateau modulus indicative of a solid network and do not all exhibit power-law behavior over extended ranges, making it difficult to unambiguously test the predicted high-frequency behavior.

In this Letter, we present the results of a study of the rheological properties of F-actin networks using diffusingwave spectroscopy (DWS) to measure the mean square displacement of probe particles in the actin network, and its frequency-dependent linear viscoelastic moduli. The use of DWS allows us to probe an extended range of frequencies, $10^{-2} \leq \omega \leq 10^{6} \mathrm{~Hz}$, in a single experiment, while ensemble averaging over a large number of independent tracer particles. We show that the data exhibit a heretofore unobserved scaling behavior; despite large variations from sample to sample, all the data for the complex modulus can be scaled onto a single master curve. This scaling demonstrates that, despite the wide variation in properties, a single parameter controls the rheology of the networks, 
particularly at the crossover from network-dominated to single-chain-dominated behavior. The scaled data also provide a convincing measure of the extended frequencydependent behavior; we find that the dominant scaling of the modulus is $G^{*}(\omega) \sim \omega^{3 / 4}$ over three decades in frequency.

Actin was purified from rabbit muscle [23] and stored in G-buffer [2 mM Tris-Cl, $0.2 \mathrm{mM}$ ATP, $0.5 \mathrm{mM}$ dithiothreitol, $\left.0.1 \mathrm{mM} \mathrm{CaCl}_{2}\right]$ at $-80^{\circ} \mathrm{C}$. The concentration of actin, $c$, was determined by UV-visible spectroscopy and by a Bradford assay [24]. Samples for DWS were prepared by thawing frozen aliquots of actin at $37^{\circ} \mathrm{C}$; phalloidin was added to a concentration of $1.2 c$ to stabilize actin filaments against depolymerization; fluorescence microscopy suggests that the resultant filaments were about $10 \mu \mathrm{m}$ long. Surface-modified polystyrene latex particles were added to a volume fraction of $1 \%$ to serve as probe particles for DWS. Polymerization of actin was induced by adding $\mathrm{MgCl}_{2}$ to a concentration of $75 \mathrm{mM}$. Prior to measurements, samples were incubated at $4{ }^{\circ} \mathrm{C}$ for 6 hours. Transmission electron micrographs showed no apparent bead aggregation or structural change of the actin network due to the presence of the tracer particles. Diffusing-wave spectroscopy $[25,26]$ was performed in the transmission geometry using samples in flat cells a few millimeters thick, and illuminated with an etalon-equipped $\mathrm{Ar}^{+}$laser at a wavelength $\lambda=514.5 \mathrm{~nm}$. Mean-square displacements $\left\langle\Delta r^{2}(t)\right\rangle$ of tracer particles were calculated by numerically inverting the measured intensity autocorrelation function using a transport mean free path, $l^{*}$, determined by measuring the transmitted light intensity relative to that of a sample of known $l^{*}$.

Typical mean-square displacements for a sample with $c \simeq 1.5 \mathrm{mg} / \mathrm{m} \ell$ are shown in Fig. 1; these data illustrate the high resolution of DWS, which is greater than single particle tracking [21,22]. At short times $\left\langle\Delta r^{2}(t)\right\rangle$ does not increase linearly in time as expected for diffusion

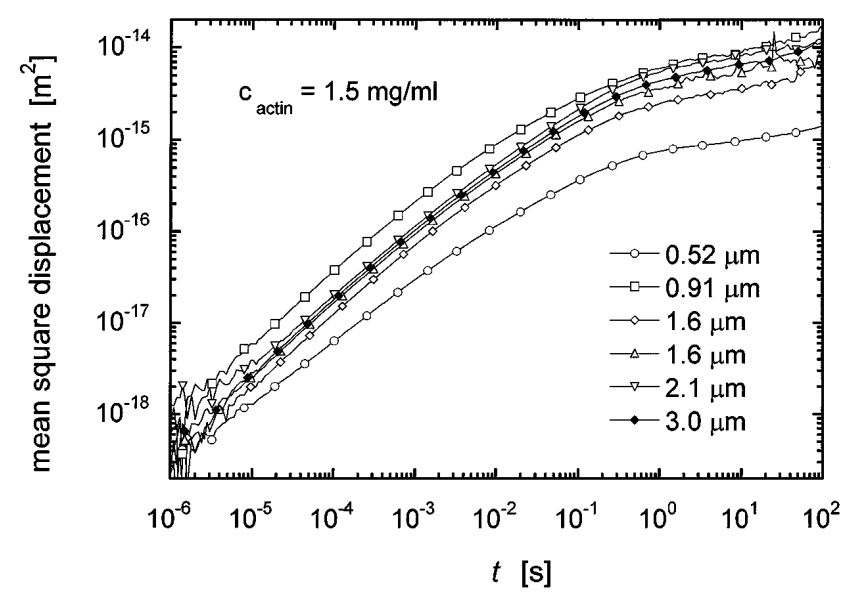

FIG. 1. Mean-square displacement $\left\langle\Delta r^{2}(t)\right\rangle$ of polystyrene latex beads in $1.5 \mathrm{mg} / \mathrm{m} \ell$ phalloidin-stabilized F-actin measured with diffusing-wave spectroscopy; symbols denote different bead sizes. in a purely viscous medium but instead increases subdiffusively, with a power law, $\left\langle\Delta r^{2}(t)\right\rangle \sim t^{\gamma}$. The value of $\gamma$ is typically $3 / 4$ but exhibits some variation from sample to sample. Furthermore, there is a rather broad crossover to a plateau at longer times where $\left\langle\Delta r^{2}(t)\right\rangle$ is only very weakly dependent on time. The plateau in the mean-square displacement at long times, $\left\langle\Delta r^{2}\right\rangle_{\mathrm{p}}$, provides a direct measure of the low-frequency elastic modulus of the network $G_{0} \simeq k_{\mathrm{B}} T /\left(\pi a\left\langle\Delta r^{2}\right\rangle_{\mathrm{p}}\right)$, where $a$ is the particle diameter. More generally, the time evolution of $\left\langle\Delta r^{2}(t)\right\rangle$ provides a measure of the frequency dependent linear viscoelastic modulus of the network [20]. The magnitude of the complex modulus is given by $G(s)=$ $k_{\mathrm{B}} T /\left(\pi a s\left\langle\widetilde{\Delta r^{2}}(s)\right\rangle\right)$, where the tilde denotes the Laplace transform, and $s$ is the Laplace frequency [20].

For a given dc modulus $G_{0}$, the maximum meansquare displacement, $\left\langle\Delta r^{2}\right\rangle_{\mathrm{p}}$, of a bead should scale as $1 / a$, provided the bead is much larger than $l_{\mathrm{m}}$; unfortunately, however, we do not observe this simple scaling, even for samples prepared with the same actin concentration. Instead, we find a very wide sample-tosample variation. Although samples prepared on the same day and at the same concentration exhibit reproducible behavior, samples prepared from different actin stocks have drastically different behavior. The magnitude of the low frequency modulus varies from sample to sample, lying between 0.1 and $10 \mathrm{~Pa}$; for some samples, the plateau behavior is not visible at all. Similarly, the value of the crossover frequency varies widely from sample to sample. Furthermore, even the apparent slope of the power-law increase at higher frequencies exhibits some variation, as does the absolute magnitude of $G(s)$. However, despite their differences, all the data do have some features in common. Most notably, the frequency range of the crossover is, in all cases, very broad; even when a plateau is not observed, there is still clearly the onset of a broad crossover.

To search for features in these data that are universal and transcend the large variations, we normalize the magnitude of the modulus by the plateau value, $G_{0}$, and the frequency by the characteristic crossover frequency, $\omega_{0}$. As shown in Fig. 2, this normalization reveals a remarkable scaling, with all the data falling together onto a single master curve, despite the very large variations from sample to sample. While there is some deviation at the highest and lowest frequency ranges observed, the scaling is remarkably good, particularly around the very broad crossover frequencies. This scaling indicates that the existence of the crossover and the very broad range of frequencies over which it occurs are robust features, universal to all actin networks.

The scaled data also provide an excellent measure of the magnitude of $G(s)$, as they are averaged over many samples; thus the full complex modulus, $G^{*}(\omega)$, can be determined accurately. We do this by fitting the average of $G(s)$ to a functional form and then making the substitution $s=i \omega$ and determining the real and imaginary parts 


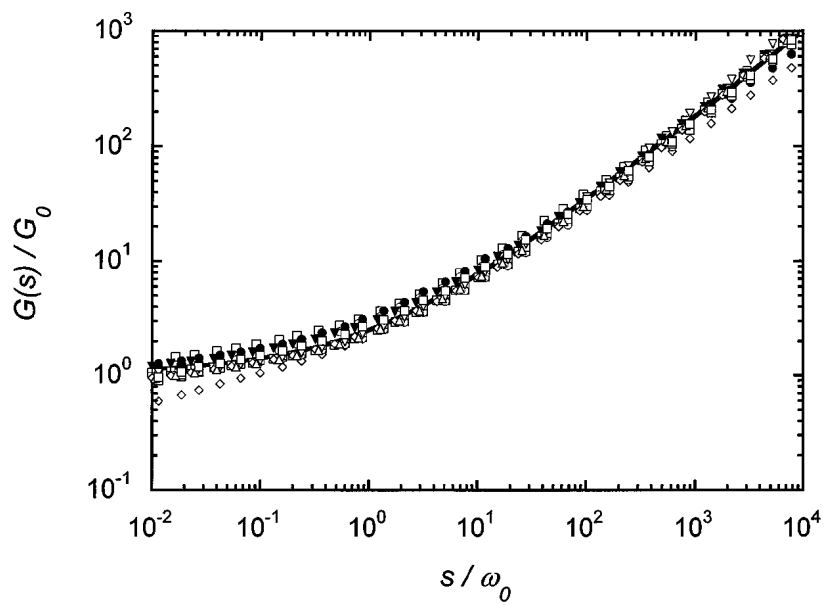

FIG. 2. $G(s)$ scaled with its low-frequency value $G_{0}$ as a function of the normalized frequency $s / \omega_{0}$. Symbols correspond to actin monomer concentrations between 1 and $2 \mathrm{mg} / \mathrm{m} \ell$ and bead diameters between 0.52 and $3 \mu \mathrm{m}$. The solid line is a fit of the ansatz $G(s) / G_{0}=1+c_{1}\left(s / \omega_{0}\right)^{1 / 3}+$ $c_{2}\left(s / \omega_{0}\right)^{3 / 4}$ to the averaged data; best-fit parameters are $c_{1}=$ 0.5 and $c_{2}=1.0$.

[20]. We fit the data to an ansatz: $G(s) / G_{0}=1+$ $c_{1}\left(s / \omega_{0}\right)^{1 / 3}+c_{2}\left(s / \omega_{0}\right)^{3 / 4}$. The addition of the second term, proportional to $\left(s / \omega_{0}\right)^{1 / 3}$, is purely empirical but is necessary to properly account for the very broad crossover. Addition of this term provides an excellent fit to the data, as shown by the solid line in Fig. 2, which is obtained using $c_{1}=0.5$ and $c_{2}=1$. The resultant values of $G^{\prime}(\omega)$ and $G^{\prime \prime}(\omega)$ are shown in Fig. 3. At low frequencies, below $\omega_{0}$, the storage modulus dominates and attains a plateau independent of frequency reflecting the solidlike behavior of the network resulting from the resistance of the configuration of entanglements to mechanical deformation [9,12]. At high frequencies $10^{2} \omega_{0} \leq \omega \leq 10^{4} \omega_{0}$, both $G^{\prime}(\omega)$ and $G^{\prime \prime}(\omega)$ scale as $\omega^{3 / 4}$; here the loss modulus dominates over the storage modulus, in agreement with

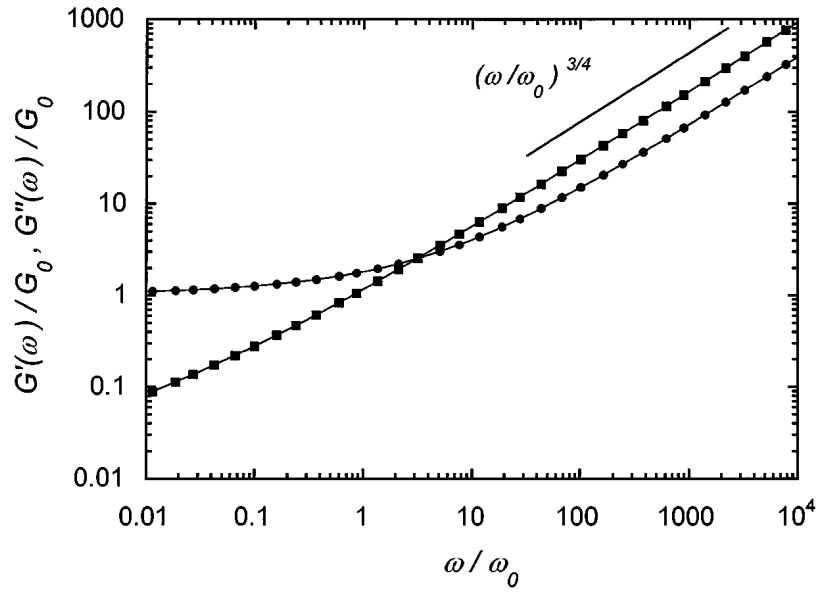

FIG. 3. Storage modulus $G^{\prime}(\omega)(\bullet)$ and loss modulus $G^{\prime \prime}(\omega)$ (ם) calculated from the light scattering data. The crossover region is broadened by a term scaling as $\omega^{1 / 3}$. theoretical predictions based on the bending dynamics of individual filaments. The crossover reflects the change in the excitations of the network; at low frequencies, the full network responds to the motion of the bead, while at high frequencies the wavelength of any excitation is shorter than the average entanglement of the filaments, and $G^{*}(\omega)$ reflects directly the single filament response. Finally, the data clearly show that the high-frequency behavior is dominated by an exponent of $3 / 4$ that extends over nearly three decades of $\omega$. We note, however, that for a few samples the exponent varies, with values ranging from 0.7 to 0.9. In addition, at the highest frequencies we expect it to approach 1 , reflecting the contribution of the viscosity of the water; however, this is never observed in our data.

Further insight comes from the scaling factors required to superimpose the data, and in Fig. 4 we plot the variation of the values of $\omega_{0}$ with $G_{0}$. Values for different actin concentrations are indicated by different symbols; there is considerable variation for data with the same nominal concentration, with both $\omega_{0}$ and $G_{0}$ varying by as much as a decade. Moreover, there is no apparent trend in the scaling parameters as the concentration is varied. This suggests that the apparent concentration of actin monomers does not determine the ultimate behavior of the network. Nevertheless, the scaling parameters do exhibit a clear correlation; although not perfect, it is best described as a linear relationship between $\omega_{0}$ and $G_{0}$, shown as the solid line in Fig. 4.

To account for this correlation, at least qualitatively, we consider a very simple model for the modulus; we assume that it is made up of two components. The first is frequency independent and reflects the contribution of the purely elastic response of the network, $G_{1}(\omega) \sim c^{\alpha}$; the second is frequency dependent and reflects the contribution of the individual filaments at high frequencies, $G_{2}(\omega) \sim c^{\beta} \omega^{3 / 4}$. The value of the exponent $\alpha$ for the

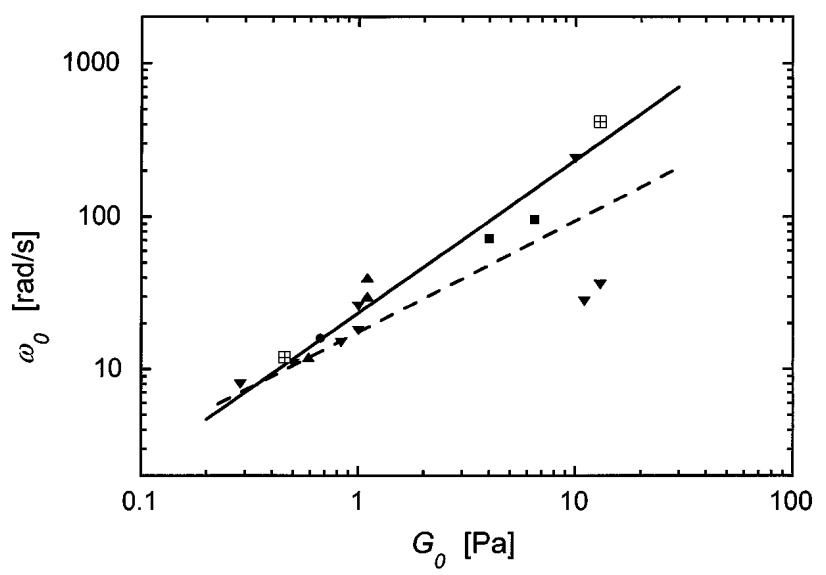

FIG. 4. Measured values of the crossover frequency $\omega_{0}$ as a function of the plateau modulus $G_{0}$; actin monomer concentrations are $0.5 \mathrm{mg} / \mathrm{m} \ell(\mathbf{O}), 1.0 \mathrm{mg} / \mathrm{m} \ell(\boldsymbol{\square}), 1.2 \mathrm{mg} / \mathrm{m} \ell(\boldsymbol{\Delta})$, $1.5 \mathrm{mg} / \mathrm{m} \ell(\boldsymbol{\nabla})$, and $2.0 \mathrm{mg} / \mathrm{m} \ell$ (田). The dashed line with a slope of 0.73 corresponds to the prediction for a strongly crosslinked network, while the solid line is a slope of 1 . 
concentration dependence of $G_{1}$ varies depending on both the existence of cross-links and the effects of the distribution of filament lengths. By contrast, $G_{2}$ depends on the properties of the individual filaments; while we expect its magnitude to reflect the number of filaments and hence depend linearly on $c$ [12], with $\beta$ we allow for a more general dependence. Because of the sampleto-sample variability, we assume that $c$ is not well determined. However, since the two components must be equal at $\omega=\omega_{0}$, we can eliminate $c$ to obtain $\omega_{0} \sim G_{0}^{z}$, where $z=4(\alpha-\beta) / 3 \alpha$.

The experimentally observed correlation implies that $\alpha \simeq 4 \beta$, a rather large value if $\beta=1$. If there are no cross-links, we expect the dc modulus to scale as $G_{1}(\omega) \sim$ $c^{\alpha}$ with $\alpha=1.4$. Such behavior has in fact been observed for very carefully purified actin [15]. However, with $\beta=1$, this yields a correlation exponent of $z=0.38$, which is well outside the uncertainty of our data. By contrast, if cross-links control the modulus, we expect $\alpha=2.2$ [9]; with $\beta=1$, this leads to a correlation exponent of $z=0.73$, shown as the dashed line in Fig. 4. While this value is less than optimal, the experimental uncertainty is sufficiently large that we cannot exclude it. However, the effect of cross-links will be most dramatic when only a very small number are added to an otherwise cross-link-free network; just two cross-links per filament will pin that filament in its tube, preventing relaxation of longitudinal fluctuations, significantly increasing the macroscopic modulus [12]. While we can not predict a value for $\alpha$ in this case, we would expect a stronger increase of $G_{0}$ with $c$, leading to a value of $z$ closer to unity. Thus, we speculate that the origin of the large variability in the modulus results from a small but random number of cross-links. The distribution of filament lengths may also play a role; shorter filaments will modify the effects of the cross-links, again resulting in a stronger variation of the modulus. This would account for the apparent insensitivity of $G_{0}$ on concentration for our actin preparations. Similar effects are implicated in the vast differences between the values of $G_{0}$ reported by different laboratories for nominally the same actin preparation and concentration [14].

Our data do not address the critical issue of the applicability of microrheology methods in determining elastic moduli that correspond to those measured with bulk methods $[12,27]$. Detailed tests of this require much greater sample-to-sample reproducibility. Nevertheless, the scaling behavior reported here does help identify the essential features of the rheology of actin networks that are universal to all conditions and does help determine the origin of the irreproducibility that commonly plagues rheological measurements of actin networks.

We thank Dennis Discher, Erwin Frey, Yale Goldman, Paul Janmey, Klaus Kroy, Margot Leonard, Fred MacKintosh, Tony Maggs, David Morse, Erich
Sackmann, Christoph Schmidt, and Sally Zigmond for helpful discussions and Paul Janmey for the electron micrographs. This work was supported by the NSF (DMR96-31279); T. G. was also supported by the Schweizerischer Nationalfonds.

*Present address: Universität Konstanz, Fakultät für Physik, P.O. Box 5560, 78457 Konstanz, Germany.

[1] M. Schliwa, The Cytoskeleton. An Introductory Survey (Springer, Wien, 1986).

[2] F. Gittes, B. Mickey, J. Nettleton, and J. Howard, J. Cell Biol. 120, 923 (1993).

[3] A. Ott, M. Magnasco, A. Simon, and A. Libchaber, Phys. Rev. E 48, R1642 (1993).

[4] J. Käs, H. Strey, M. Bärmann, and E. Sackmann, Europhys. Lett. 21, 865 (1993).

[5] K. C. Holmes, D. Popp, W. Gebhard, and W. Kabsch, Nature (London) 347, 44 (1990).

[6] K. Kroy and E. Frey, Phys. Rev. Lett. 77, 306 (1996).

[7] R. Satcher and C. Dewey, Biophys. J. 71, 109 (1996).

[8] D. E. Ingber, Sci. Am. 278, No. 1, 48 (1998).

[9] F. C. MacKintosh, J. Käs, and P. A. Janmey, Phys. Rev. Lett. 75, 4425 (1995).

[10] H. Isambert and A.C. Maggs, Macromolecules 29, 1036 (1996).

[11] A. C. Maggs, Phys. Rev. E 55, 7396 (1997).

[12] D. C. Morse, Phys. Rev. E 58, R1237 (1998).

[13] F. Gittes and F. C. MacKintosh, Phys. Rev. E 58, R1241 (1998).

[14] J. Xu, W.H. Schwarz, J. A. Käs, T.P. Stossel, P. A. Janmey, and T. D. Pollard, Biophys. J. 74, 2731 (1998).

[15] B. Hinner, M. Tempel, E. Sackmann, K. Kroy, and E. Frey, Phys. Rev. Lett. 81, 2614 (1998).

[16] F. Amblard, A. C. Maggs, B. Yurke, A. N. Pargellis, and S. Leibler, Phys. Rev. Lett. 77, 4470 (1996).

[17] A. Ajdari, R. Everaers, F. Jülicher, and A.C. Maggs, report.

[18] K. S. Zaner and P. A. Valberg, J. Cell Biol. 109, 2233 (1989).

[19] F. Ziemann, J. Rädler, and E. Sackmann, Biophys. J. 66, 2210 (1994).

[20] T. G. Mason and D. A. Weitz, Phys. Rev. Lett. 74, 1250 (1995).

[21] F. Gittes, B. Schnurr, P.D. Olmsted, F. C. MacKintosh, and C. F. Schmidt, Phys. Rev. Lett. 79, 3286 (1997).

[22] T. G. Mason, K. Ganesan, J. H. van Zanten, D. Wirtz, and S. C. Kuo, Phys. Rev. Lett. 79, 3282 (1997).

[23] J. D. Pardee and J. A. Spudich, Methods Enzymol. 85, 164 (1982).

[24] J. Sambrook, E. F. Fritsch, and T. Maniatis, Molecular Cloning. A Laboratory Manual (Cold Spring Harbor Laboratory Press, Plainview, NY, 1989), 2nd ed.

[25] G. Maret and P. E. Wolf, Z. Phys. B 65, 409 (1987).

[26] D. J. Pine, D. A. Weitz, P. M. Chaikin, and E. Herbolzheimer, Phys. Rev. Lett. 60, 1134 (1988).

[27] A. C. Maggs, Phys. Rev. E 57, 2091 (1998). 